\title{
HITCal: a software tool for analysis of video head impulse test responses
}

\author{
JORGE REY-MARTINEZ ${ }^{1}$, ANGEL BATUECAS-CALETRIO ${ }^{2}$, EUSEBI MATIÑO $^{3}$ \& \\ NICOLÁS PEREZ FERNANDEZ ${ }^{4}$
}

${ }^{1}$ Otolaryngology Unit ORLGipuzkoa, Clinica Quirón, San Sebastián, ${ }^{2}$ Otoneurology Unit, ENT Department, IBSAL, University Hospital of Salamanca, Salamanca, ${ }^{3}$ Department of Otolaryngology, Hospital General de Catalunya, San Cugat Del Vallés, Barcelona and ${ }^{4}$ Department of Otolaryngology, Clínica Universidad de Navarra, Pamplona, Spain

\begin{abstract}
Conclusion: The developed software (HITCal) may be a useful tool in the analysis and measurement of the saccadic video head impulse test (vHIT) responses and with the experience obtained during its use the authors suggest that HITCal is an excellent method for enhanced exploration of vHIT outputs. Objective: To develop a (software) method to analyze and explore the vHIT responses, mainly saccades. Methods: HITCal was written using a computational development program; the function to access a vHIT file was programmed; extended head impulse exploration and measurement tools were created and an automated saccade analysis was developed using an experimental algorithm. For pre-release HITCal laboratory tests, a database of head impulse tests (HITs) was created with the data collected retrospectively in three reference centers. This HITs database was evaluated by humans and was also computed with HITCal. Results: The authors have successfully built HITCal and it has been released as open source software; the developed software was fully operative and all the proposed characteristics were incorporated in the released version. The automated saccades algorithm implemented in HITCal has good concordance with the assessment by human observers (Cohen's kappa coefficient $=0.7$ ).
\end{abstract}

Keywords: Algorithm, head impulse test, fitter, saccades analysis, vestibular physiology, vHIT, VOR

\section{Introduction}

The assessment of the vestibulo-ocular reflex (VOR) with the use of a new video-based tool that measures the eye velocity in response to fast, high acceleration, and unpredictable head impulses, in different planes, also called the video head impulse test (vHIT) is increasingly becoming a standard for the evaluation of patients with dizziness. It allows for the evaluation of each of the receptors in the different semicircular ampullae of the canals [1].

In any normal subject a fast head thrust to one side will evoke an eye movement that matches the former in velocity; the eye movement occurs in the opposite direction in such a way that at the end of the head movement the eye remains in place because both displacements were similar (Figure 1). The amounts of head and eye velocity are characterized by their quotient, also called 'gain' of the VOR. In the case when the head movement is incorrectly detected, as occurs in patients with vestibulopathy, the eye movement will be under-compensatory, and an eye position error ensues that provokes visual blurring. To resolve this situation, fast eye movements will be produced to move the eye to the expected end point: these are called refixation saccades (Figure 1).

The very initial (occurring $<100 \mathrm{~ms}$ ) eye movement after the head impulse is mainly driven by the stimulation and corresponding depolarization of the hair cells in the vestibular receptor; this information travels to the corresponding ocular motor nuclei across three synapses. The late refixation saccades can occur early, while the head is still in movement (covert saccades) or once the head stops (overt saccades), and central nervous system structures are responsible for their generation. The early covert saccades are triggered by 


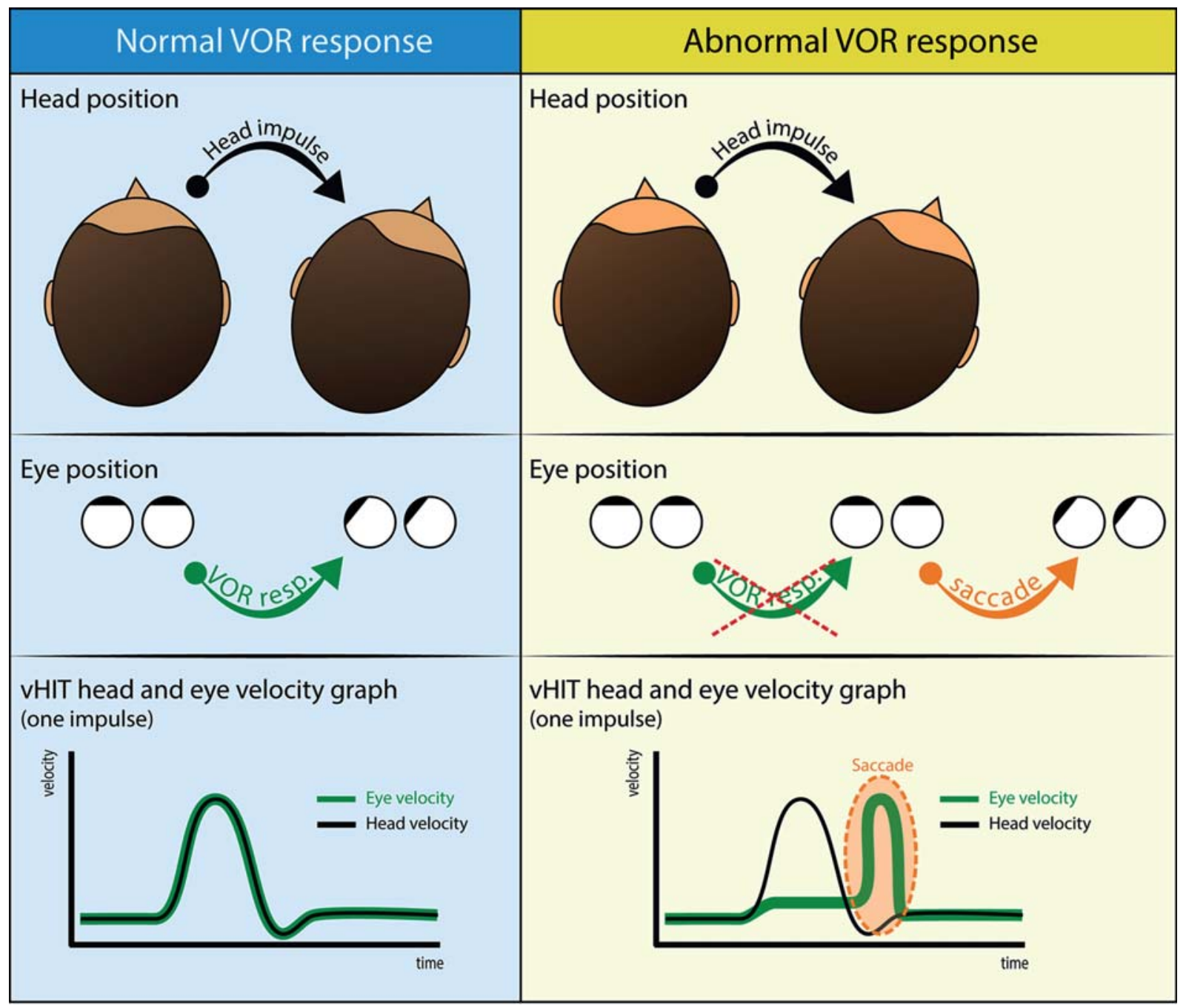

Figure 1. Recording vestibulo-ocular reflex (VOR) responses. With normal VOR function eye movements follow the head movement in reverse direction (left column). With abnormal VOR function eye movements do not follow head movement and refixation saccades are produced (right column). The video head impulse test (vHIT) plots the graphs of the head (black line) and eye (green line) motion as seen at the bottom of the figure. The data plotted in this figure with the vHIT head and eye motion are an idealized representation, these graphics do not represent real data plots.

the vestibular input; otherwise the late overt saccades are visually guided saccades [2]. The horizontal saccadic movements originate in the superior colliculus in the midbrain and project the information, first to the nucleus reticularis and pontine reticular formation, and then to the abducens nucleus. The vertical saccadic movements originate in the rostral interstitial nucleus of the medial longitudinal fasciculus and in the interstitial nucleus of Cajal [3].

With the use of a head-mounted camera to register the eye movement and a sensor for head velocity, the gain of the VOR is available for assessment.

Different protocols for the evaluation of gain have been developed, some of which analyze the relation of velocities at different periods of time after the head impulse or the area under the curve for both head and eye velocity for an extended period of time. However, saccades have been poorly reported other than by their appearance, although they are of major interest for clinical evaluation of the compensating patient [4].

The objective of this study was to develop and test an extended method for exploration of vHIT responses, with special focus on refixation saccade analysis. For this purpose a computer software program named 'HITCal' was created with the process described hereinafter.

To date, only a few works have carried out a deep study with the parametric characteristics of the saccades [5]; unfortunately, the methodology used in them to measure the saccades was not published as an open access methodology. With the aim of giving a methodology that can be further easily used by other 
researchers, the results of our research will be published as an open source project.

It is important to remark that HITCal was not developed to substitute the actual vHIT analysis methods. HITCal is a not official extension to the ICS-vHIT (ICS impulse, GN Otometrics, Taastrup, Denmark) software that uses part of the standard ICSvHIT outputs to give a new interpretation of the vHIT results. This extended interpretation of the vHIT results is a method that has not been reported before based on the quantification of the refixation saccades.

\section{Material and methods}

This section will be divided into two parts: the development process of HITCal and the laboratory testing of the saccades analysis method implemented in HITCal.

\section{HITCal development}

HITCal was written using the computational development program MATLAB (MATLAB Release 2013b, The MathWorks, Inc., Natick, MA, USA). The following steps were carried out.

1. Accessing a vHIT file. This was the first step: to create a function to read the vHIT software (ICSDatabase version 1.2.23, ICS impulse, GN Otometrics) results files. By default the vHIT software allows three file formats for data export: (1) XML (eXtensible Markup Language) format, (2) CSV (CommaSeparated Values), and (3) the RAW (non-processed data) format. We choose the XML format for use in HITCal from which the functions to navigate inside the XML file were written. The access method for the XML file recognition was written in MATLAB code; this method goes over the XML file and stores all impulse data with some metadata information (such as patient's name, direction of impulse, ICS-vHIT calculated gain of all the impulses in the same plane, and calculated gain for each impulse in the same plane). With the metadata information a very intuitive navigation tool was the first interface function in HITCal; this menu shows the patient name and the type of test selected.

One important issue with the XML format is that in the XML files the vHIT data are stored in time samples, according to the ICS-vHIT technical specification we assumed a constant frame rate of 250 frames per second (fps). This frame rate was used to define our sample unit in graphics and was used as the default fame rate in all HITCal calculations.

2. Impulse exploration and measuring area. The exploration and measuring functions are among the main features of HITCal. The exploration of the HIT is shown in two plots (Figure 2). The first plot shows all the impulses performed in the selected test, one by one, and a very simple algorithm marks a saccade (if it

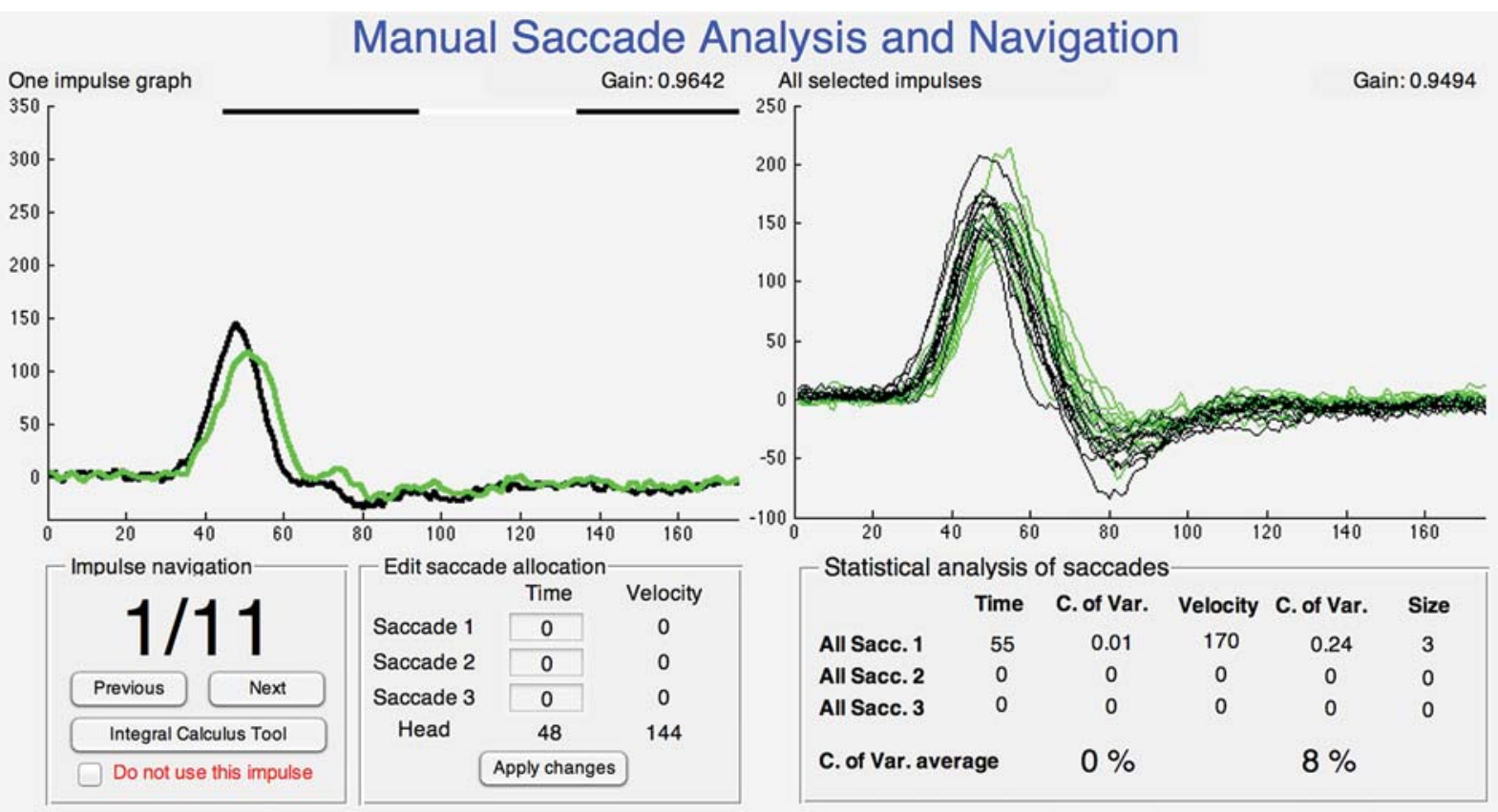

Figure 2. HITCal impulse exploration and measuring area. This area is divided into two sections; in the first section HITCal plots the records of the impulse test one by one, and in the second area HITCal plots all the selected impulses of the full test. In these graphs HITCal gives navigation options, saccade manual selection tools, integral calculus of head and eye movements, and some statistical parameters of the responses. 
occurs) in section 1 , section 2 , and section 3 , according to the time after the initiation of the impulse at which the saccade occurs; the user can manually obtain the time and the velocity data of any point in the head or eye velocity graphs and modify the given results. The second plot shows all the impulses from the selected test. Under this plot, HITCal shows some statistical data obtained from the saccades (as selected by the system or manually) in each section: the mean and the coefficient of variation for the time at which the saccades occur and their velocity. An integral calculus tool designed to obtain the head and eye positions and their acceleration is also presented in this section [RN 1].

3. Automated saccade analysis method. The purpose of this part is to characterize the level of organization of the saccades that occur after several head impulses in one direction, based on previous work that has shown that well organized or isochronically appearing saccades after head impulses (Figure 3) are seen in patients with a well-compensated vestibulopathy, and disorganized or non-isochronically appearing saccades (Figure 3) are more frequently seen in uncompensated patients [6]. A MATLAB code algorithm based on the Jitter parameter solution similar to that used to determine the clustering of some complex signals, as sound [7], was developed to analyze the saccades organization. This algorithm was specifically designed to analyze the HITs of patients with no acute vestibular dysfunction; our algorithm is not designed to compute spontaneous nystagmus.

A necessary function to reach an algorithm that characterizes the organization of the saccades is a system to recognize the saccades in the eye records of the vHIT. The method used in point 2 of this section (Impulse exploration and measuring area) uses a simple algorithm that finds the maximum values of the eye velocity in three pre-established time intervals. This method could be appropriate to assist the user when setting the saccades manually, but we consider that it is not valid as an automatic method to detect the saccades. A new method was designed using the MATLAB function findpeak, this MATLAB function finds local maxima from an input data (see MATLAB reference guide) and was implemented in our algorithm using the following arguments. Minpeakdistance, used to set the minimum distance between two peaks, with this argument we can establish an interval of time after our saccade in which no other saccade will be detected. Minpeakheight, used to set the minimum value for a saccade, with this argument we can depreciate low velocity saccades or artifacts in the vHIT measurements. Npeaks, used to determine the number of peaks on the data array, this argument is especially useful to detect the peak in the head impulse movement data, where only one peak must be found, this peak in the head impulse movement was used to determine the initial time sample where the function begins to search the saccades in the eye movement. With this initial point in the eye data we ensured that no saccades were detected before (and 10 samples after) the head peak impulse.

The authors of HITCal did not find bibliographic references to determine the values of these arguments; to solve this a sample of 20 real vHITs selected by an experiment observer were manually measured using MATLAB, the impulses were only selected by the experiment observer if a significant number of saccades were detected on them. The collected data were

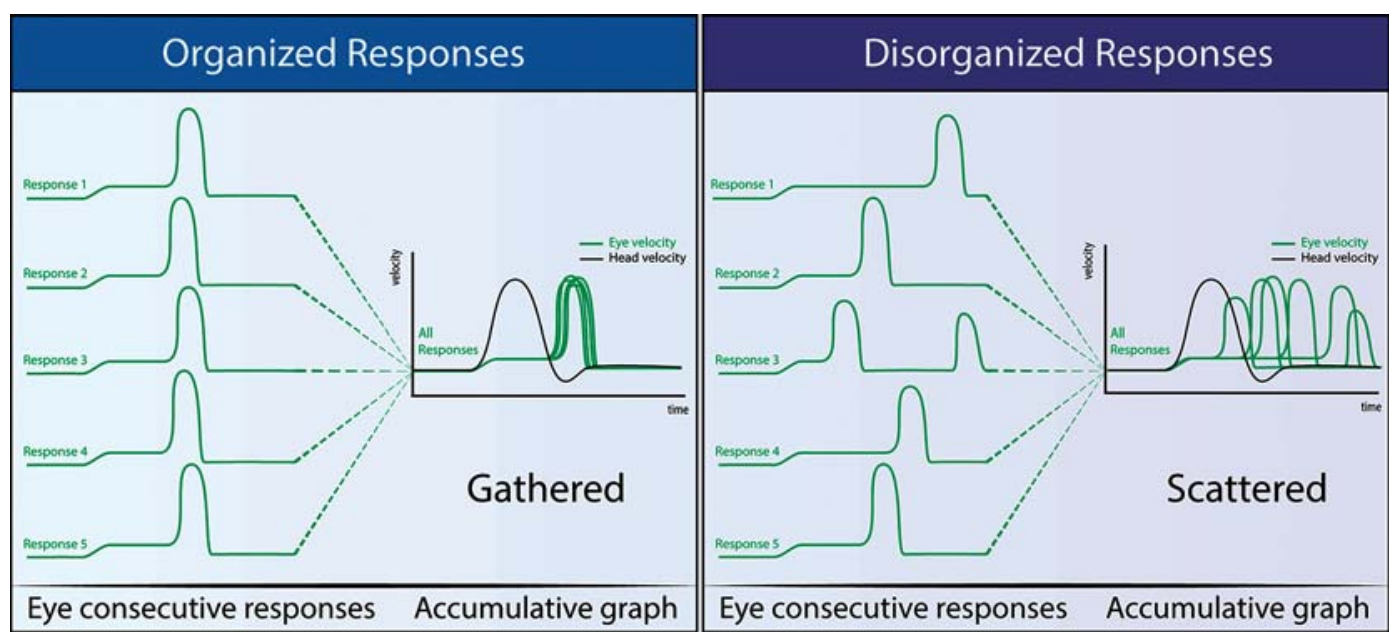

Figure 3. Organized and disorganized saccade concept. Accumulated responses on repetitive head impulses produce saccades with a similar time delay (organized responses) or with a variable time delay (disorganized responses). The data plotted in this figure with the vHIT head and eye motion are an idealized representation, these graphics do not represent real data plots. 
used to determine the arguments of the findpeak function finally implemented in HITCal.

A second correction was applied to the detected saccades. If the appearance of any saccade was located outside of $a+20$ or -15 time interval (distance in samples from the mean of appearance of all detected saccades), these saccades were marked as outliers. Only the first two of these outlier saccades were unmarked as saccades.

After the time and velocity of the peaks of each saccade had been detected the next step was to organize the saccades by type in order to analyze the saccades of the same type together. In many other vHIT studies the saccades were classified as overt and covert [2] in a physiopathologic way. This is a very useful classification, but we found a main issue if we used this classification in our algorithm. A covert saccade is defined as an eye response that occurs during the head movement; this time interval (from the peak of the head movement to the end of the head movement) is a short time interval. All the (covert) saccades that occur in this short time interval necessary must appear in the same (or very near) moment of time. What is the sense of measuring the grouping of the overt saccades if they are gathered by definition?

We decided to classify the saccades only in attendance to the order of appearance during the eye response. All the detected saccades were stored in the first, second, third, and fourth saccades groups.

The final task in the algorithm is to output a magnitude that informs the user about the level of organization of the saccades. For this, the PR score (and a PR algorithm) was developed. The authors of this study adopted the 'PR' denomination by consensus and this denomination does not have any mathematical or scientific significance. The PR score was designed as a magnitude between 0 and 100, where 0 is a minimum scattered saccadic HIT and 100 is a maximum scattered saccadic HIT. To get the PR score, the first coefficient of variation for each saccade time of appearance for each group of saccades were calculated, with this a first, second, third, and fourth saccades PR score were obtained. To calculate the 'global' PR score a weighted arithmetic mean was calculated with first saccades PR score and second saccades PR score ( 0.8 of first PR score +0.2 of second PR score). The following corrections were applied to the calculated mean. First the mean was multiplied by 2.5 , this correction was necessary to increase the magnitude of the PR score and make its maximum values to the upper limit of 100; if the result was over 100 the value was reset to 100 . A second correction was applied to underestimate the values obtained with the HITs in which a low number of saccades were detected; this correction decreases the weight of the second group saccades in the arithmetic mean inversely to the number of saccades detected. This second correction was only applied if the calculated PR score was over 35. HITCal finally presents the value obtained with these corrections to the user as the PR score.

The algorithm finally implemented in HITCal, called the 'PR algorithm' is summarized in Table I. The PR algorithm has two main outcomes, one categorical with three patterns, normal, gathered or scattered, and the 'PR score,' a numeric score with the aim of measuring the degree of scattered responses present in the analyzed HIT.

Table I. Automated saccade analysis algorithm.

\begin{tabular}{|c|c|c|}
\hline & Saccade recognition sequence & PR score algorithm \\
\hline Step 1 & $\begin{array}{l}\text { Low pass filter of the head and eye graphs (the low pass filter } \\
\text { algorithm and specific parameters are published in the } \\
\text { 'low.m' file) }\end{array}$ & $\begin{array}{l}\text { Pattern classification logic condition: } \\
\text { If coefficient of variation of eye peak }>12 \text { for first saccades } \\
\text { group or coefficient of variation }>35 \text { for second saccades } \\
\text { group. Result is scattered }\end{array}$ \\
\hline Step 2 & $\begin{array}{l}\text { Relative max values recognition, using conditions: } \\
\text { Minimum velocity: } 65^{\circ} / \mathrm{s} \\
\text { Minimum distance between peaks in eye graph: } 15 \text { samples } \\
\text { Minimum distance with head velocity peak: } 10 \text { samples }\end{array}$ & $\begin{array}{l}\text { Group PR score: Coefficient of variation of peaks in each } \\
\text { group } \\
\text { The group is determined by the order of appearance of } \\
\text { saccades }\end{array}$ \\
\hline Step 3 & $\begin{array}{l}\text { Outlier detection, only the two first peak values with }+20 \text { or } \\
-15 \text { difference from mean of peaks were unmarked as } \\
\text { saccades }\end{array}$ & $\begin{array}{l}\text { Global PR score calculation: } \\
2.5^{\star}\left(0.8^{\star} \mathrm{CV} 1+0.2^{\star} \mathrm{CV} 2\right) \\
\text { Where CV1 is coefficient of variation of first registered } \\
\text { saccades and CV2 is coefficient of variation of second } \\
\text { registered saccades } \\
\text { Global PR score corrections: } \\
\text { If score is }>100 \text {, the value is reset to } 100 \\
\text { If score is }>35 \text {, the value is adjusted with the number of } \\
\text { peaks detected: if only a few impulses have peaks over } 35 \text {, } \\
\text { these impulses have a low impact on the PR score }\end{array}$ \\
\hline
\end{tabular}

The main steps in saccade recognition sequence and the PR score algorithm are shown. For time samples a frame rate of 250 fps is used. 

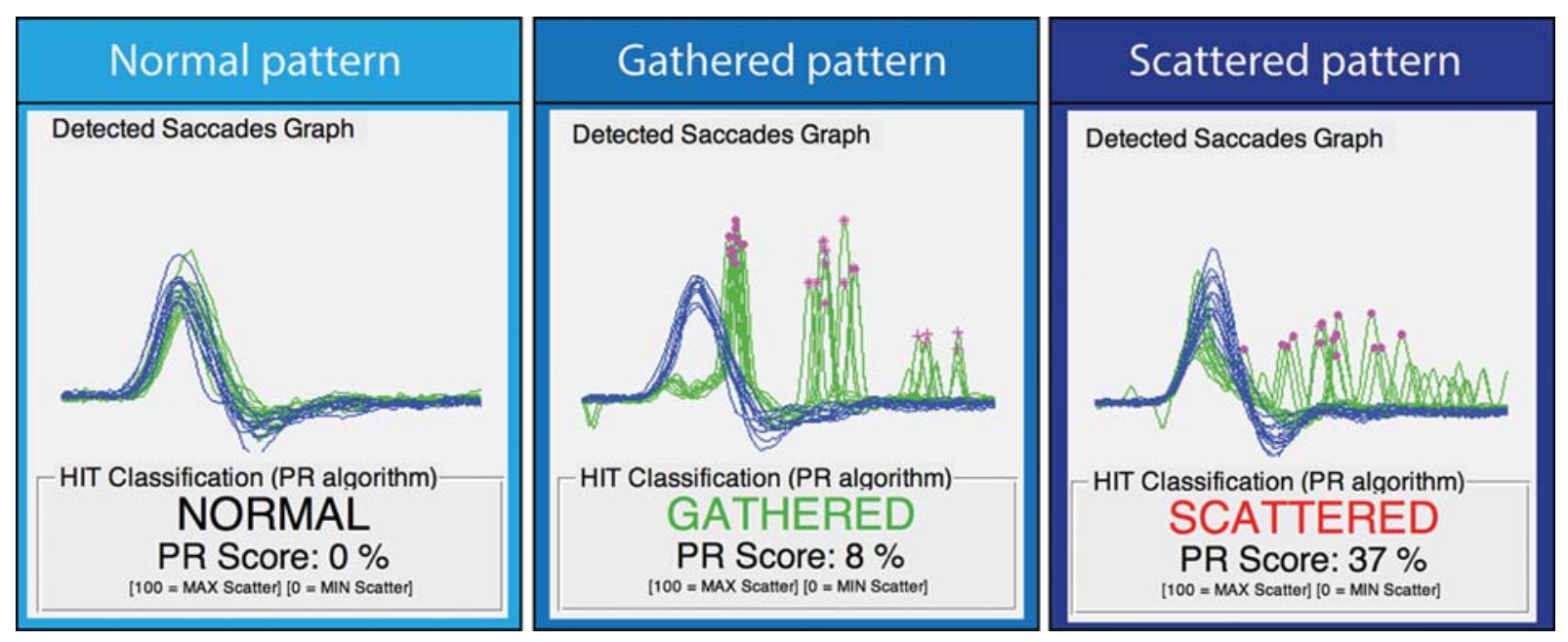

Figure 4. HITCal 'automated saccade analysis' output. Normal pattern, no saccades were detected; scattered pattern, disorganized or nonisochronically appearing saccades after head impulses were detected; gathered pattern, well organized or isochronically appearing saccades after head impulses were detected. The PR score increases with more disorganized saccades.

The final version of the automated saccade analysis system incorporated into HITCal is shown in Figure 4.

\section{HITCal testing}

For HITCal saccades analysis pre-release laboratory tests, a database of HITs was created. Registers obtained at three different hospitals were used for this evaluation; in each, a physician experienced in the use and interpretation of the vHIT was in charge of the collection of data.

Data from 10 patients were obtained from each hospital. Patients were selected if saccades were seen after head impulses delivered to one or both sides in the horizontal plane; patients with spontaneous nystagmus (to either side) or saccades in the same head impulse direction were excluded.

A fourth investigator collected the data and resent each investigator the other investigators' selected tests, requiring them to provide a classification for each impulse test. The requested classification had two parameters. The first parameter was the pattern of clustering for each impulse test, the pattern values were 'normal,' 'gathered' or 'scattered.' The second parameter was the level of organization of the saccades, ranked from 1 to 10 , where 1 means no scattered responses and 10 indicates maximum scattered responses.

The fourth investigator built the final 30-test database with the other investigators' responses. With this crossed methodology each investigator never evaluated the impulses that they originally sent to the fourth investigator.
Then, the XML files exported from all selected HITs were presented to HITCal, and the computed results were incorporated in the investigators' response database.

For the saccades pattern the kappa index was calculated, and the Pearson's correlation was calculated with the degree of scattering for the saccades; SPSS 22.0.0 (IBM Corp.) for Macintosh was used for statistical analysis.

Data for this study were stored in a file as anonymous content (identifiable patient information was not included in the database used in this study). These terms are in accordance with Spanish regulation applicable to the protection of personal data (LOPD 15/1999).

The design of this retrospective study was carried out following the guidelines of the Declaration of Helsinki of the World Medical Association on Ethical Principles for Medical Research Involving Human Subjects. The Ethics Committee of each participating hospital accepted the protocol of this study.

\section{Results}

As a result of the HITCal development process a stand-alone application was compiled using MATLAB compiler; this software itself is a main result of our research. The application was fully operative in the computers used, and there were no problems processing the XML files generated with the supported ICS-vHIT files (ICSDatabase version 1.2.23). The main functions of HITCal software are: impulse selection from the XML vHIT files, standard and advanced impulse plots with 'data 


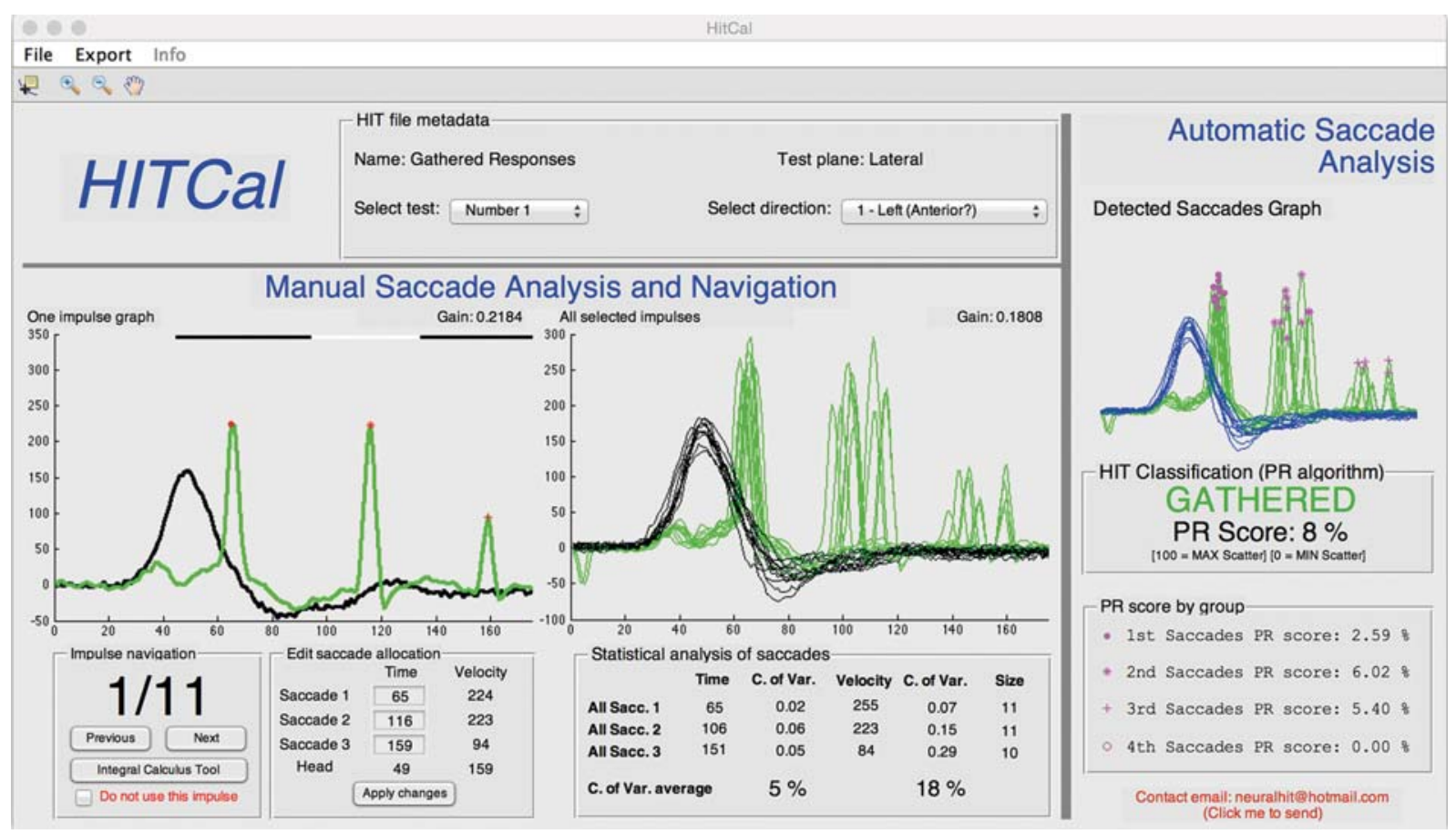

Figure 5. HITCal. View of the final HITCal main window running on OS X Yosemite (10.10) Macintosh system (C Apple Inc.).

cursor' mode, manual saccade recognition, and automatic saccade analysis. For this automatic saccade analysis a new methodology called the PR score was developed. The final developed version of HITCal software is shown in Figure 5.

The HITCal software, all the support files and the source code used in its development were released as an open source project on the web (http://mlibra. com).

PR score ranking and a categorical pattern to characterize the level of organization of the saccades were programmed in HITCal. The entire PR algorithm that contains all these methodologies is contained between the code lines 1057 and 1281 of the file 'HitCal.m' published in the mlibra.com website. The aims of this open source project are to support the results of this study and to stimulate other researchers to continue developing this (or similar) vHIT projects.

As results for the laboratory test performed with HITCal, data from 30 patients aged 21-59 years were included in this retrospective study. Their mean age was 36.2 years $(\mathrm{SD}=6$ years); 16 patients were male and 14 were female. The concordance study gave a mean HITCal-Observer Cohen's kappa coefficient of 0.7 and a mean of Pearson's correlation of 0.8. The results of the laboratory concordance tests are detailed in Table II.

\section{Discussion}

Our interest in focusing the software on the refixation saccades analysis is because this is an integral part of the complete VOR evoked after head impulses that stimulate the vestibular receptor. They occur as a consequence of a deficient VOR to minimize retinal smear [4]. In their generation, other structures and functions, mainly visual and neck reflexes and preprogramming, are influential. The objective of this software is to facilitate some quantitative analysis and qualitative description as to the level of organization of the response. This is a

Table II. HITCal pre-release test results.

\begin{tabular}{lcccccc}
\hline Statistical test $(n=20)$ & $\mathrm{HC}$ vs O1 & $\mathrm{HC}$ vs O2 & $\mathrm{HC}$ vs O3 & O1 vs O2 & O1 vs O3 & O2 vs O3 \\
\hline Kappa coefficient & 0.65 & 0.81 & 0.89 & 0.93 & 0.70 & 0.63 \\
Pearson's correlation & 0.85 & 0.90 & 0.73 & 0.92 & 0.71 & 0.60 \\
\hline
\end{tabular}

Cohen's kappa coefficient and Pearson's correlation between HITCal saccade analysis and human observer responses. HC, HITCal; O1, human observer 1; O2, human observer 2; O3, human observer 3. In all results $p<0.01$. In each HITCal vs human, $n=20$; in each human vs human, $n=10$. 
very dynamic response, as can be seen in the followup of patients with severe vestibular damage in the short term [8] or in the long term during vestibular compensation [5].

The first step in the development of HITCal was the selection of the vHIT exported file format to use. XML was selected because it is a structured file with a multitude of useful metadata that includes some HIT parameters such as gain, max velocity, number of impulses, and the numerical data of the results of the impulses, and also because it saves the results impulse by impulse. Using XML format we acquire the HIT results processed with the vHIT software algorithms. The CSV format is good for spreadsheet software importation, but there is not much metadata stored in this file format and there is no structured and labeled data storage such as found in XML. RAW files save complete results, needing a previous step for the detection of each impulse.

One of the main limitations of our tool is that a constant sample rate of 250 fps was assumed; however, the data stored in the XML file are not always constant as the ICS-vHIT software automatically rejects low sample rates to minimize registration artifacts. As such this 'near to' 250 sample rate could affect the HITCal outputs. The ICS-vHIT fabricant recommends using the RAW data file for some calculations; in this RAW file the real time is registered for each measurement. The problem with this RAW format is that all the data recorded during the test are included. The RAW file is a large record file, inside which all events are recorded: the eye and head movements that occurred during the well-performed impulses, the impulses that were not performed well, and even any other movement that occurred during the entire test time. To design an automated analysis method based on RAW files, a new impulse recognition algorithm is needed. This algorithm must analyze the entire RAW data selecting the wellperformed impulses. The implementation of this impulse recognition algorithm exceeds the proposed methodology for this study. To solve this, we decided to use the XML file format; in which all the data stored are preprocessed by the ICS vHIT software algorithm to select the well-performed impulses.

The other main issue in HITCal was that many of the parameters used in the algorithms are based on our in-house manual measures of saccades. This was the best way that we found to develop some parts of HITCal; until the date of the HITCal first release (January 2014) the authors did not find any published data about the main parameters that characterize the saccadic movements in the eye response, apart from its appearance and the covert and overt classification. It was necessary to measure many parameters such as expected peak velocity, minimum velocity required to consider an eye response as a saccade, number of possible saccades that can occur in one eye response, time delay between saccades that occur in the same eye response, and similar parameters during the HITCal development process. It is important to remark that many of these measurements were performed using preliminary versions of HITCal (without an operative automatic saccades analysis algorithm), so our own HITCal provides us with the possibility of working with a very user-friendly and powerful tool to analyze the HIT head and eye movements. The authors consider that investigations to obtain a deeper knowledge about the saccades will be mandatory in the future. This idea is in accordance with other authors that have recently reported the clinical relevance of an improved analysis of the saccadic responses in the HIT [9]; we hope that HITCal could help to achieve this goal.

The use of the system was off-line and was found to be easy after some trials. We selected a period of time to acquire the predetermined number of tests for statistical analysis. In the analysis of the variable 'degree of scattering,' Pearson's correlation was performed instead of intraclass correlation coefficient. First, because we cannot assume that human observers measure this variable with the same scale as HITCal, and second, because, for a correct use of intraclass correlation coefficient, the tested variables must have the same measure scale outputs as Fisher and others previously mentioned [10,11], unavailable in our work.

With the known limitations of the pre-release tests performed with HITCal, the low number of cases and the statistical method used that we discussed before, the automated saccades algorithm implemented in HITCal has good concordance with the evaluations by human observers [12].

Another important point about this work is that all HITCal files are published as an open source project. Apart from the advantages previously discussed, this provides the possibility for any other researchers to contribute to this software tool, with their own improvements (based on further investigations) applied to the PR algorithm or to any other part of this software.

These results demonstrate that HITCal may be a useful tool in the measurement of saccadic vHIT responses, and with the experience obtained during its use the authors suggest that HITCal is an excellent method for enhanced exploration and measurement of vHIT outputs. 
Declaration of interest: The authors report no conflicts of interest. The authors alone are responsible for the content and writing of the paper.

\section{References}

[1] Curthoys IS. The interpretation of clinical tests of peripheral vestibular function. Laryngoscope 2012;122:1342-52.

[2] Weber KP, Aw ST, Todd MJ, McGarvie LA, Curthoys IS, Halmagyi GM. Head impulse test in unilateral vestibular loss: vestibulo-ocular reflex and catch-up saccades. Neurology 2008;70:454-63.

[3] Delgado-Garcia JM. Why move the eyes if we can move the head? Brain Res Bull 2000;52:475-82.

[4] Mcdougall HG, Curthoys IS. Plasticity during vestibular compensation: the role of saccades. Front Neurol 2012;3:21.

[5] Heuberger M, Sağlam M, Todd NS, Jahn K, Schneider E, Lehnen N. Covert anti-compensatory quick eye movements during head impulses. PLoS One 2014;9:e93086.

[6] Batuecas-Caletrio A, Santa Cruz Ruiz S, Muñoz Herrera A, Perez Fernandez N. The vestibulo-ocular reflex and

\section{Reference Notes}

1. Rey-Martinez J, Perez-Fernandez N, Ispizua A, Urrutikoetxea A, Matino E, et al. (2014) How to apply classical mechanics to the results of the video head impulse test? J Otolaryngol ENT Res 2014; 1(1): 00004. DOI: 10.15406/joentr.2014.01.0000 subjective balance after vestibular schwannoma surgery. Laryngoscope 2014;6:1431-5.

[7] Iriarte J, Fernández S, Fernandez-Arrechea N, Urrestarazu E, Pagola I, Alegre M, et al. Sound analysis of catathrenia: a vocal expiratory sound. Sleep Breath 2011;15: 229-35.

[8] Mantokoudis G, Schubert MC, Tehrani AS, Wong AL, Agrawal Y. Early adaptation and compensation of clinical vestibular responses after unilateral vestibular deafferentation surgery. Otol Neurotol 2014;35:148-54.

[9] Chen L, Todd M, Halmagyi GM, Aw S. Head impulse gain and saccade analysis in pontine-cerebellar stroke and vestibular neuritis. Neurology 2014;83:1513-22.

[10] Bravo G, Potvin L. Estimating the reliability of continuous measures with Cronbachs alpha or the intraclass correlation coefficient: towards the integration of two traditions. J Clin Epidemiol 1991;44:381-90.

[11] Fisher RA. On the "probable error" of a coefficient of correlation deduced from a small sample. Metron 1921;1: $1-32$.

[12] Altman DG. Practical statistics for medical research. New York: Chapman and Hall; 1991. 
Copyright of Acta Oto-Laryngologica is the property of Taylor \& Francis Ltd and its content may not be copied or emailed to multiple sites or posted to a listserv without the copyright holder's express written permission. However, users may print, download, or email articles for individual use. 